\title{
Caracterización y evaluación de la sustentabilidad de fincas cafetaleras en la localidad de Nuevo Chirimoto, Rodríguez de Mendoza-Región Amazonas
}

\section{Coffee farm characterization and evaluation of its sustainability in Nuevo Chirimoto, Rodríguez de Mendoza - Amazonas Region}

\author{
Zoila R Guevara $\mathrm{M}^{1}$. y Rosario E Vásquez $\mathrm{C}^{2}$.
}

\begin{abstract}
El objetivo del trabajo fue caracterizar y evaluar la sustentabilidad de fincas de café. Se trabajó con la Asociación de productores agropecuarios "Flor de Café" conformada por 351 agricultores. Se realizó una encuesta teniendo en cuenta aspectos económicos, sociales y ambientales, el 100\% de la producción de café es orgánica, con sistemas de cultivo con escasa asociación y diversificación de cultivos, además existe una alta dependencia por este tipo de cultivo. Se aplicó también el método multicriterio utilizando indicadores de tipo económico, ecológico y sociocultural, el aspecto económico el $54 \%$ de las fincas no son sustentables, debido a la poca o nula variedad de productos teniendo como consecuencia ingreso netos mensuales bajos. La dimensión ecológica resultó ser más sustentable (97\%), esto se debería a que hacen un manejo de cobertura vegetal conducentes a la protección del suelo contra la erosión. En el aspecto sociocultural, la sustentabilidad alcanzada fue del $84 \%$ y esto sería por el fácil acceso a los servicios básicos de salud y educación. El índice de general determinó que el $44 \%$ de fincas presentaron un índice $\geq 2$, lo que las convierte en fincas sustentables.
\end{abstract}

Palabras clave: caracterización, finca, sustentabilidad, café, indicadores.

\begin{abstract}
The objective of the work was the characterization and evaluation of the sustainability of coffee farms. We worked with the Association of agricultural producers "Flor de Café" made up of 351 farmers. A survey was carried out considering economic, social and environmental aspects, $100 \%$ of coffee production is organic, with cultivation systems with little association and crop diversification, there is also a high dependence on this type of crop. The multicriteria method was also applied using economic, ecological and sociocultural indicators, the economic aspect 54\% of the farms are not sustainable, due to the little or no variety of products resulting the low monthly income. The ecological dimension turned out to be more sustainable (97\%), this is due to the fact that they manage vegetation cover leading to soil protection against erosion. In the socio-cultural aspect, the sustainability achieved was $84 \%$ and this would be due to the easy access to basic health and education services. The general index determined that $44 \%$ of farms had an index $\geq 2$, which makes them sustainable farms.
\end{abstract}

Keywords: characterization, coffee farms, Sustainability, Indicators 


\section{INTRODUCCIÓN}

El café es el principal producto agrícola de exportación en el Perú, según la Junta Nacional del Café (JNC) en el 2013 indica que este se produce en 338 distritos de 16 departamentos. La superficie cultivada con café ocupa 415,000 hectáreas, distribuidas en tres zonas, siendo la región más apropiada para obtener los mejores rendimientos con alta calidad la que se ubica al extremo central oriental de la Cordillera de los Andes, en la denominada zona de la selva, bajo una ecología tropical. Según la información obtenida del MINAGRI (2013a) las regiones que lideran la producción de café son Junín, San Martín, Cajamarca, Cusco y Amazonas de las que se obtiene más del $90 \%$ de la producción nacional de café.

La producción de café en el departamento de Amazonas ha ganado importancia social y económica, las condiciones ambientales favorables con las que cuenta para el cultivo de esta especie le permite producir un café de calidad, las provincias productoras de café son Luya, Rodríguez de Mendoza, Utcubamba, cuyas características competitivas radican en que son café de altura, se siembran bajo sombra las variedades que se siembran son Typica, Caturra y Bourbón (MINAGRI, 2003b). La provincia de Rodríguez de Mendoza, donde se llevó a cabo el presente estudio se caracteriza por su producción orgánica de café $(70 \%)$ con características organolépticas que lo califica como un producto de excelente calidad, pero en la cadena productiva se presentan diversos problemas que influye en el rendimiento y afecta el ingreso de los productores, esto conlleva a implementar proyectos de mejora tecnológica las mismas que deben llevarse a cabo bajo los criterios de una agricultura sostenible. Una característica importante radica en la caracterización del sistema como un paso previo a cualquier proyecto posterior y así aprovechar las potencialidades propias y las oportunidades del entorno con el objetivo de incrementar su productividad, rentabilidad, competitividad y sostenibilidad. Martos (2004) dice que la caracterización es una etapa determinante para el desarrollo del método de investigación en sistemas de producción, es un análisis descriptivo que nos permite, además, elaborar un marco de estudio a partir del cual se deduce una problemática o bien formular un diagnóstico con el fin de conocer carencias esenciales y sugerir una acción posterior. Según Velarde, y Quiroz (1994), la caracterización a nivel de finca permite clasificar la función que cumple cada componente de los sistemas en relación al todo y permite la generación y difusión de alternativas tecnológicas.

Por otro lado, el concepto de sustentabilidad ha cobrado cada vez importancia; actualmente, se ha convertido en uno de los elementos clave para el manejo de recursos naturales. Según Daly (2002) la sustentabilidad se puede entender como el desarrollo que satisface las necesidades de las generaciones presentes sin comprometer la habilidad de las generaciones futuras de satisfacer las suyas. Sarandón (2002), por su parte sostiene que la sustentabilidad es un concepto complejo porque pretende cumplir con varios objetivos en forma simultánea que involucran dimensiones 
productivas, ecológicas o ambientales, sociales, culturales, económicas y fundamentalmente temporales. La sustentabilidad evaluada en este trabajo se llevó a cabo aplicando un enfoque cualitativo bajo la utilización de indicadores propuestos por Sarandón(2002). Los objetivos del presente estudio fueron caracterizar y evaluar la sustentabilidad de las fincas cafetaleras de la localidad de Nuevo Chirimoto, Amazonas, Perú.

\section{MATERIALES Y MÉTODOS}

El estudio se realizó en la localidad de Nuevo Chirimoto, distrito de Omia, provincia de Rodríguez de Mendoza, departamento de Amazonas, Perú. Geográficamente se ubica entre los paralelos $06^{\circ}$ $28^{\prime} 04^{\prime \prime}$ de latitud sur y entre los meridianos $77^{\circ} 23^{\prime} 45^{\prime \prime}$ de longitud oeste con una altitud de 1363 msnm. El promedio de precipitación anual es 1005.70 ( $\mathrm{mm} / \mathrm{año})$, la temperatura media anual es de $20.9{ }^{\circ} \mathrm{C}$. Para la investigación se eligió a la Asociación de Productores Agropecuarios "Flor de Café" que tiene un total de 351 productores, de esta población se obtuvo una muestra $(n=78)$ que fue estimada utilizando el "método de las proporciones" metodología propuesta por INCAGRO, recomendada por Julca et al. (2009) y citada en Merma \&Julca (2011). La metodología aplicada para el desarrollo de la investigación es como se describe:

a) Caracterización: La obtención de la información de cada una de las fincas consistió en administración de encuestas que incluyó cuestionarios semi estructurados cuyos resultados fueron analizados y tabulados utilizando el paquete Microsoft Excel.

b) Evaluación de la sustentabilidad: se aplicó el método multicriterio utilizando indicadores para las dimensiones económico, ecológico y socio-cultural los mismos que se construyeron utilizando la metodología propuesta por Sarandón (2002) el cual sigue los lineamientos de Smyth y Dumansky (1995) y Astieret al. (2002). El objetivo de los indicadores es proveer una base empírica y numérica para conocer los problemas, calcular el impacto de nuestras actividades en el medio ambiente y para evaluar el desempeño de las políticas públicas (Rodríguez, 2002). Los indicadores fueron estandarizados en una escala numérica (0 a 4), siendo 0 la categoría menos sustentable y 4 la más sustentable. Los indicadores fueron ponderados multiplicando el valor de la escala por un coeficiente de acuerdo a la importancia relativa de cada variable con respecto a la sustentabilidad, por lo tanto, el peso de cada valor refleja la importancia del mismo en la sustentabilidad. Los indicadores fueron agrupados de acuerdo a su naturaleza y dimensión de análisis como se indica en la tabla 1.

Se estimó la sustentabilidad general (IS general) de las fincas cafetaleras promediando los valores obtenidos para las tres dimensiones. Según Sarandónet al. (2004), una finca es sustentable siel valor de IS General es mayor a 2 y ninguna de las tres dimensiones deben tener un valor $<2$ 
Tabla 1. Indicadores de sustentabilidad para las fincas cafetaleras de la localidad de Nuevo Chirimoto según la metodología de Sarandón (2002).

\begin{tabular}{|c|c|c|c|c|c|c|c|}
\hline \multirow{2}{*}{$\stackrel{\Xi}{\Xi}$} & \multirow{2}{*}{ indicadores } & \multicolumn{5}{|c|}{ escala de estandarización } & \multirow{2}{*}{$\begin{array}{c}\text { Factor } \\
\text { de } \\
\text { ponder. }\end{array}$} \\
\hline & & $\mathbf{0}$ & 1 & 2 & 3 & 4 & \\
\hline \multicolumn{8}{|c|}{ dimensión económica } \\
\hline A1 & $\begin{array}{l}\text { Diversificación de la } \\
\text { producción }\end{array}$ & $<2$ prod. & 2 a 3 prod. & 4 a 6 prod. & 7 a 8 prod. & $>9$ prod. & 1 \\
\hline $\mathrm{A} 2$ & $\begin{array}{l}\text { superficie de producción de } \\
\text { autoconsumo }\end{array}$ & $<0.5$ has. & $\begin{array}{l}0.5 \text { a } 1 \\
\text { has. }\end{array}$ & 1.1 a 1.5 has. & 1.6 a 2 has. & $>2$ has. & 1 \\
\hline $\mathrm{B}$ & $\begin{array}{l}\text { Ingreso neto mensual (en } \\
\text { soles) }\end{array}$ & $<300$ & 300 a 499 & 500 a 699 & 700 a 999 & $>1000$ & 2 \\
\hline $\mathrm{C} 1$ & diversificación para la venta & 1 prod. & 2 prod. & 3 prod. & 4 a 6 prod. & $>6$ prod. & 1 \\
\hline $\mathrm{C} 2$ & $\begin{array}{l}\text { Número de vías de } \\
\text { comercialización }\end{array}$ & 1 canal & 2 canales & 3 canales & 4 canales & $\begin{array}{c}>5 \\
\text { canales }\end{array}$ & 1 \\
\hline $\mathrm{C} 3$ & $\begin{array}{c}\text { Dependencia de insumos } \\
\text { externos }\end{array}$ & 0 a $20 \%$ & 21 a $40 \%$ & 41 a $60 \%$ & 61 a $80 \%$ & $\begin{array}{c}81 \mathrm{a} \\
100 \% \\
\end{array}$ & 1 \\
\hline \multicolumn{8}{|c|}{ Dimensión ecológica } \\
\hline A1 & $\begin{array}{l}\text { Manejo de la cobertura } \\
\text { vegetal }\end{array}$ & $<25 \%$ & 25 a $50 \%$ & 51 a $75 \%$ & 76 a $99 \%$ & $100 \%$ & 1 \\
\hline $\mathrm{A} 2$ & rotación de cultivos & Ninguna & $\begin{array}{l}\text { rotaciones } \\
\text { eventuales }\end{array}$ & $\begin{array}{l}\text { cada } 203 \\
\text { años }\end{array}$ & $\begin{array}{l}\text { todos los } \\
\quad \text { años }\end{array}$ & $\begin{array}{l}\text { dejando } \\
\text { un año }\end{array}$ & 1 \\
\hline A3 & Diversificación de cultivos & $\begin{array}{l}\text { Monocult } \\
\text { ivo }\end{array}$ & $1 \mathrm{cv}$ & $2 \mathrm{cv}$ & $3 \mathrm{cv}$ & $>4 \mathrm{cv}$ & 1 \\
\hline B1 & Pendiente predominante & $>45 \%$ & $\begin{array}{l}30.1 \text { a } \\
45 \%\end{array}$ & 15.1 a $30 \%$ & 5.1 a $15 \%$ & 0 a $5 \%$ & 2 \\
\hline B2 & cobertura vegetal & 0 a $24 \%$ & 25 a $49 \%$ & 50 a $74 \%$ & 75 a $99 \%$ & $100 \%$ & 1 \\
\hline B3 & orientación de los surcos & $\begin{array}{l}\text { paralelos } \\
\text { a la } \\
\text { pendiente }\end{array}$ & $\begin{array}{c}60^{\circ} \\
\text { respecto a } \\
\text { la } \\
\text { pendiente }\end{array}$ & $\begin{array}{l}\text { contra la } \\
\text { pendiente }\end{array}$ & $\begin{array}{c}\text { perpendicula } \\
\text { r a la } \\
\text { pendiente }\end{array}$ & $\begin{array}{c}\text { curvas a } \\
\text { nivel de } \\
\text { terraza }\end{array}$ & 2 \\
\hline $\mathrm{C} 1$ & Biodiversidad espacial & $\begin{array}{l}\text { Monocult } \\
\text { ivo }\end{array}$ & $1 \mathrm{cv}$ & $2 \mathrm{cv}$ & $3 \mathrm{cv}$ & $>4 \mathrm{cv}$ & 1 \\
\hline $\mathrm{C} 2$ & Biodiversidad temporal & Ninguna & $\begin{array}{l}\text { rotaciones } \\
\text { eventuales }\end{array}$ & $\begin{array}{c}\text { cada } 203 \\
\text { años }\end{array}$ & $\begin{array}{l}\text { todos los } \\
\text { años }\end{array}$ & $\begin{array}{l}\text { dejando } \\
\text { un año }\end{array}$ & 1 \\
\hline \multicolumn{8}{|c|}{ Dimensión sociocultural } \\
\hline A1 & Acceso a la educación & $\begin{array}{c}\sin \\
\text { acceso }\end{array}$ & Primaria & Secundaria & técnico & $\begin{array}{c}\text { universit } \\
\text { ario } \\
\text { bien }\end{array}$ & 1 \\
\hline $\mathrm{A} 2$ & $\begin{array}{l}\text { acceso a la salud y cobertura } \\
\text { sanitaria }\end{array}$ & $\begin{array}{l}\text { sin centro } \\
\text { sanitario }\end{array}$ & $\begin{array}{l}\text { centro mal } \\
\text { equipado y } \\
\text { sin } \\
\text { personal }\end{array}$ & $\begin{array}{l}\text { centro mal } \\
\text { equipado y } \\
\text { personal } \\
\text { temporal }\end{array}$ & $\begin{array}{l}\text { medianamen } \\
\text { te equipado } \\
\text { con personal } \\
\text { temporal }\end{array}$ & $\begin{array}{l}\text { equipado } \\
\text { con } \\
\text { personal } \\
\text { permane } \\
\text { nte }\end{array}$ & 2 \\
\hline A3 & calidad de vivienda & muy mala & Mala & Regular & buena & $\begin{array}{c}\text { muy } \\
\text { buena }\end{array}$ & 2 \\
\hline A4 & acceso a servicios básicos & 1 servicio & 2 servicios & 3 servicios & 4 servicios & $\begin{array}{l}>4 \text { s. } \\
\text { Básicos }\end{array}$ & 2 \\
\hline B1 & $\begin{array}{l}\text { Aceptabilidad del sistema de } \\
\text { producción }\end{array}$ & $\begin{array}{l}\text { Insatisfec } \\
\text { ho }\end{array}$ & $\begin{array}{c}\text { poco } \\
\text { satisfecho }\end{array}$ & $\begin{array}{l}\text { medianamente } \\
\text { satisfecho }\end{array}$ & satisfecho & $\begin{array}{c}\text { muy } \\
\text { satisfech } \\
\text { o }\end{array}$ & 2 \\
\hline $\mathrm{C} 1$ & Integración social & Nula & Baja & Media & alta & muy alta & 1 \\
\hline D1 & $\begin{array}{l}\text { Conocimiento y conciencia } \\
\text { ecológica }\end{array}$ & Nula & Baja & Media & alta & muy alta & 1 \\
\hline
\end{tabular}




\section{RESULTADOS Y DISCUSIÓN}

\section{Caracterización del productor y de las fincas cafetaleras}

Del resultado de la encuesta se encuentra que las personas responsables de las fincas son mayormente de sexo masculino (62.82\%) cuyas edades se encuentran comprendidas entre 31 a 40 años $(39.74 \%)$ y la población mayor a 51 años coinciden, con un $17.95 \%$. Si en cierta manera la población > 51 años es un indicador de experiencia, pero son menos propensos a adoptar nuevas tecnologías y menos proclives a capacitarse. El nivel de instrucción de los productores de café es muy variado, el 60.26\%tienen educación primaria, hay un grupo significante que tienen estudios superiores que va desde el nivel técnico hasta el universitario (6.41\%), el 33.33\% tienen educación secundaria. Buttel et al. (1990) sostiene que los agricultores con estudios completos o superiores tienden a tener un mejor acceso a la información y pueden tener mejores probabilidades de adoptar nuevas tecnologías de diferentes tipos. El $41.03 \%$ de los productores tienen un ingreso mensual menor a S/. 300 soles, el 17.95\% tienen un ingreso entre S/. 501-700 soles y solamente un 7.69\% tienen un ingreso mayor a S/. 1500 nuevos soles. Referente a las vías de acceso a su localidad se puede ver que el $76.92 \%$ tiene acceso a carretera y el $23.08 \%$ cuentan con camino de herradura. La principal actividad económica en la zona es la agricultura $(80.77 \%)$ pero tambien se dedican a la ganadería paralelo con la agricultura (19.23\%). El acceso a los servicios básicos de las fincas el $70.89 \%$ de la población encuestada consume agua entubada y el $29.11 \%$ lo consume de otras fuentes (acequia, manantial, etc.), asimismo el servicio de desagüe tambien es deficiente el $85.09 \%$ no cuentan con este servicio y los que cuentan (14.10\%) viven generalmente cerca del centro poblado. La gran ventaja es que el $84.62 \%$ cuentan con luz eléctrica. El $39.74 \%$ no han recibido capacitaciones en los últimos dos años, el 52.56\% requieren capacitaciones en control de plagas, seguido del $25.64 \%$ que requieren en manejo integral de café, el 10.26\% solicitan capacitaciones en aumento de la producción de café y un $3.85 \%$ en comercio internacional. El 84.62\% no cuentan con título de propiedad de sus terrenos, y solamente el $15.38 \%$ si cuentan con título de propiedad. 

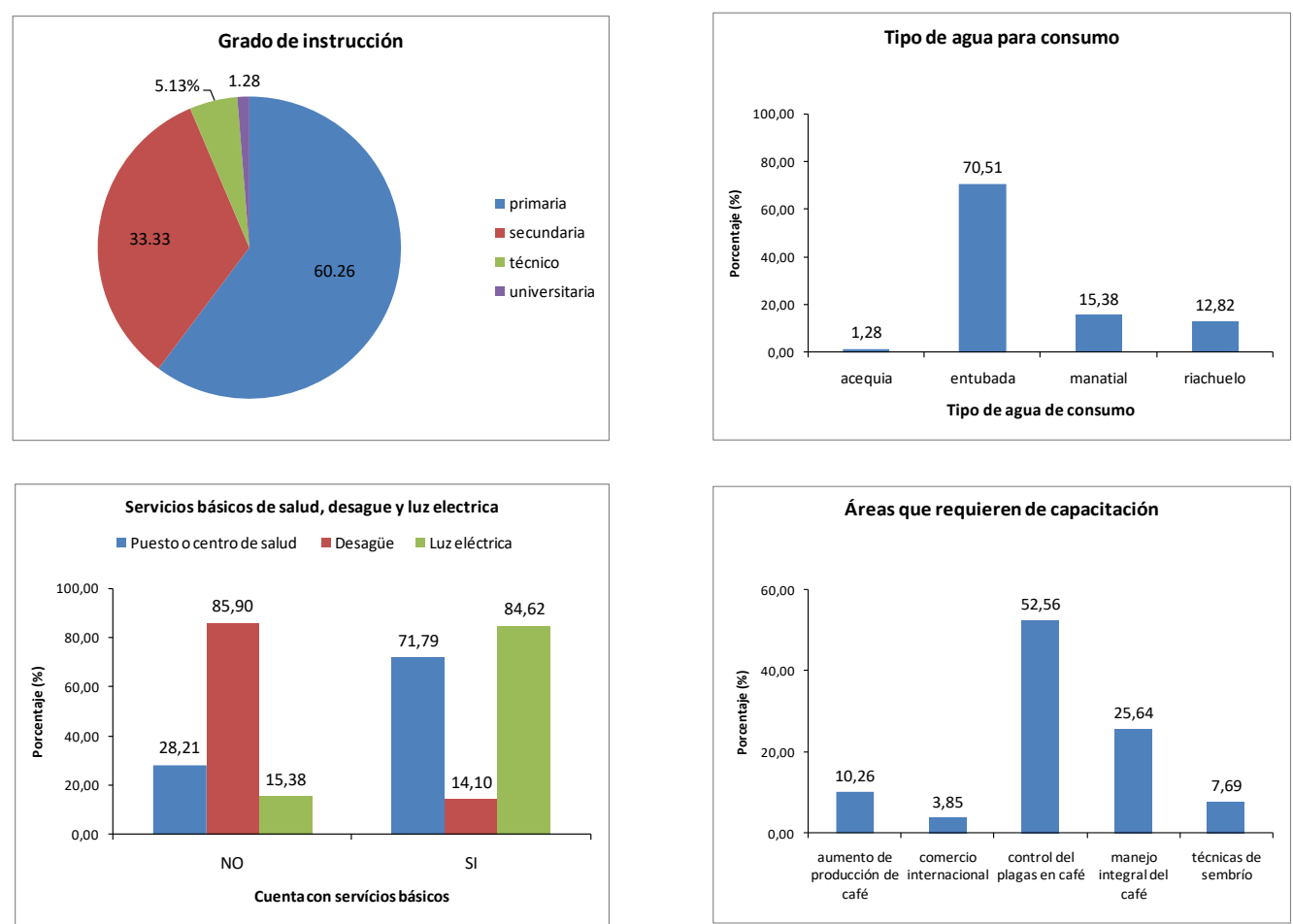

Figura 1. Nivel de instrucción, actividad económica, servicios básicos (agua, desagüe, luz), capacitaciones requeridas. Los datos están en porcentajes.

\section{Características de la producción cafetalera}

Según se muestra en la figura 2, el tipo de café que se cultiva en la zona de estudio pertenece mayormente a la variedad catimor $(42.31 \%)$, el $37.18 \%$ cultivan la variedad típica y catimor, el $15.38 \%$ solamente cultiva típica, y un grupo pequeño de agricultores cultivan la variedad caturra (2.56\%). El 100\% cultivan café orgánico y lo venden en seco. El área de la finca sembrada con café, revela que los encuestados $(76.92 \%)$ posee menos de 5 hectáreas donde prácticamente casi la totalidad están sembradas de café $(98.72 \%)$ el $14.10 \%$ poseen entre 6 a 10 hectáreas, pero solamente el $1.28 \%$ están sembradas de café. El café mayormente lo cultivan bajo sombra (94.87\%) donde la variedad de árbol más común que utilizan es el árbol de la Huaba. Algunos autores citados por Farfán (2007), señalan que la importancia y el efecto de las interacciones entre café y el árbol de sombra, depende de las condiciones del lugar, del genotipo de café y la especie arbórea a usar, y también del manejo del cultivo de café. Aparte de café el $67.95 \%$ de los cafetaleros poseen otros cultivos diferentes al café, como yuca, árboles frutales, plátano, maíz yucas además de criar animales menores cuya producción es mayormente para el autoconsumo que compensa el bajo nivel de ingresos de los productores. La inversión en la finca oscila entre S/. 1001 a S/. 5000 por hectárea (50\%) y el 38.46\% invierte menos de S/. 1000 soles. La inversión se destina principalmente para fertilización, jornal y la cosecha, no hay otro costo importante en el manejo del cultivo porque el 100\% de los encuestados no utiliza plaguicidas. El 
rendimiento de café promedio es de 21 qq/hectárea. Los lugares donde se realiza la comercialización de café son a las cooperativas (78.21\%) y a los intermediarios $(21.79 \%)$, las cooperativas representan a las asociaciones cafetaleras donde los encuestados pertenecen. El precio de venta por quintal oscila entre 300 a 320 soles $(69.23 \%)$ y 341 a 360 soles $(29.49 \%)$.
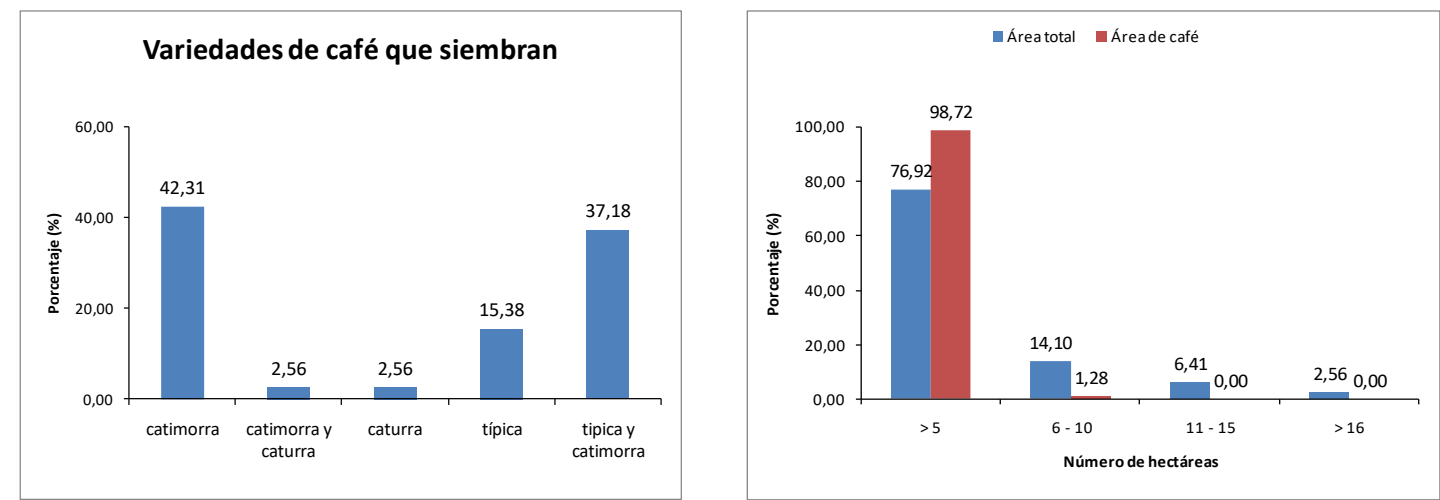

Figura 2.- Variedad de café que siembran, área total y área de café sembrado, comercialización del café. Los datos están en porcentajes.

\section{Factores ambientales de la finca}

La fuente de abastecimiento del agua procede del agua de lluvia (75.64\%) y el $24.36 \%$ utilizan agua de río, este se convierte en un factor limitante cuando existe escasez de agua e influye en la producción. El $66.67 \%$ fertiliza con abono orgánico, mientras que el $33.33 \%$ no aplica fertilizante. El abono que utilizan mayormente es roca fosfatada y guano de la isla, pulpa de café y rastrojos orgánicos. Dentro de las plagas más comunes que atacan a sus cultivos se encuentran la roya, la broca del café y el ojo de gallo. El $67.25 \%$ se sienten feliz con la producción de su finca, el $26.92 \%$ poco satisfecho y el $5.13 \%$ se sienten muy feliz.
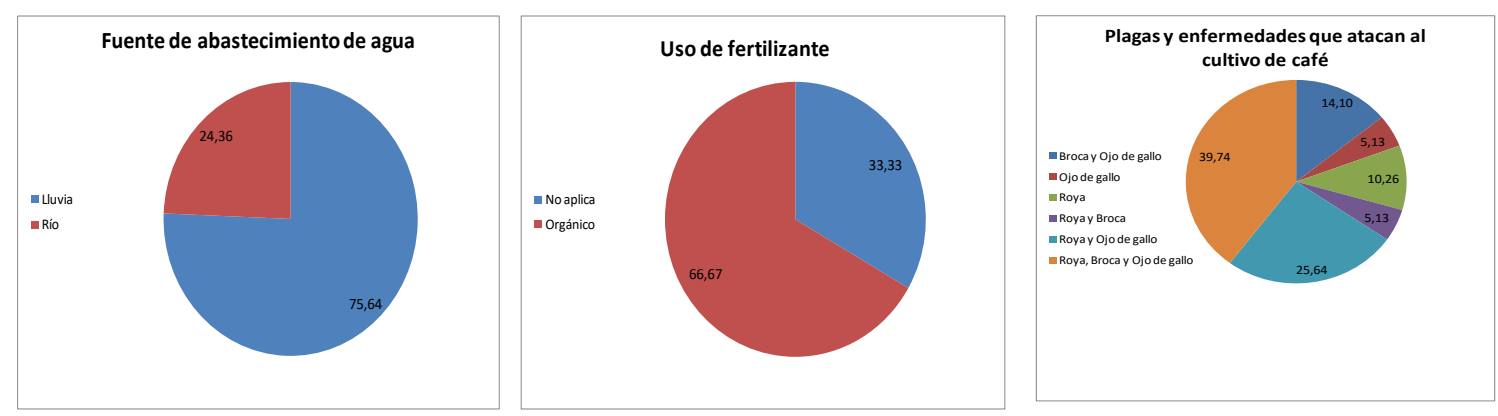

Figura 3.- Fuente de abastecimiento de agua, uso de fertilizantes, plagas que atacan al cultivo.

\section{Sustentabilidad de las fincas de café.}

Dimensión económica: los resultados muestran que el $46 \%$ de las fincas son económicamente sustentables $(\geq 2)$ y el $54 \%$ de las fincas presentan una situación crítica para 
la sustentabilidad $(\leq 2)$. Esto explica en que tienen poca diversificación productiva y tener no más de dos canales de comercialización, a pesar que presentan una superficie de producción destinada al autoconsumo por encima de las 0.5 ha y una nula dependencia de insumos externos.

Dimensión ecológica: Los resultados indican que el 97\% de las fincas son sustentables. Una de las condiciones para considerar un sistema ecológicamente sustentable es la conservación del capital natural (Constanza \&Daly, 1992, Harte 1995). De acuerdo con el análisis de sustentabilidad realizado 76 fincas cumplirían con este criterio, manteniendo la biodiversidad, la vida del suelo y evitando la erosión. La sustentabilidad ambiental aplicada a la agricultura está referida a la capacidad de garantizar la continuidad de la productividad agraria mediante el uso de prácticas en favor del uso adecuado de los recursos naturales (Gómez-Limón \& Arriaza, 2011), este alto porcentaje se debe principalmente a la baja dependencia de insumos externos como insecticidas, pesticidas, asimismo aplican un buen manejo de cobertura vegetal conducente a proteger el suelo, cultivan bajo el sistema agroforestal, pero no practican la rotación de cultivos. Altieri (2002) considera que la rotación de cultivos entre las parcelas en algunos casos aporta nutrientes, provee de fuentes de materia orgánica diversa, y disminuye la incidencia de problemas sanitarios, entre otros aspectos. En este sentido, un sistema es sustentable a medida que disminuye el periodo de rotación a lo largo del tiempo.

Dimensión socio-cultural: Los indicadores sociales están orientados a evaluar la satisfacción del productor, su calidad de vida y la integración social (Sarandónet al., 2004). Los resultados muestran que el $84 \%$ de las fincas son sustentables, fundamentalmente porque tienen satisfecho lo servicios básicos, acceso a la educación y a la salud donde el $77 \%$ tienen acceso a un puesto de salud implementado y con personal permanente. El $71 \%$ de los agricultores están satisfechos con la producción de su finca.

Índice de sustentabilidad general (ISgeneral): Según Sarandónet al. (2004), una finca es sustentable si el valor de IS General es mayor a 2 y ninguna de las tres dimensiones deben tener un valor $<2$. De acuerdo a este enunciado, el índice de sustentabilidad promedio de las 78 fincas fue 2.17, aunque se observaron diferencias entre los valores de las diferentes dimensiones. En promedio, el manejo de las fincas satisfizo en mayor grado la dimensión ecológica (2.49), que los socioculturales (2.24) y económicos (1.78). En este estudio, solamente 34 fincas cumplieron con los requisitos para ser consideradas sustentables, que equivale al $44 \%$ y 44 fincas $(56 \%)$ no cumplieron con la condición requerida para ser consideradas como sustentables. Estos resultados se explican principalmente por factores 
económicos, como es la variabilidad del precio, la poca diversificación y el bajo ingreso mensual que obtienen.

La evaluación de la sustentabilidad es un objetivo difícil de alcanzar debido a la propia complejidad del término. El uso de indicadores, a través de un análisis multicriterio puede resultar un instrumento válido para traducir esta complejidad en valores objetivos y claros que permitan cuantificar y comparar estos aspectos (Sarandón, 2002). Exige la necesidad de emplear un enfoque sistémico y holístico con una óptica multicriterio para abordar la multidimensión de la sustentabilidad (Mendoza \&Prabhu, 2000).

\section{REFERENCIAS BIBLIOGRÁFICAS}

Altieri M. 2002. Agroecología: Bases científicas para una agricultura sustentable. Guaíba, BR. Agropecuaria, 592p.

Astier M; López S; PérezE; Macera O. 2002. El marco de evaluación de sistemas de manejo incorporando indicadores de sustentabilidad (MESMIS) y su aplicación en un sistema agrícola campesino en la Región Purhepecha, Mexico. En Agroecología: el camino hacia una agricultura sustentable. Ediciones Científicas Americanas. 250 p.

ButtelF.; O. Larsonand G.W. Gillespie, JR. 1990. The New Sociology of Agriculture II: The Environment of Agriculture. In: The Sociology of Agriculture. New York: Greenwood Press, pp. 129-170.

Costanza R y Daly H. 1992. Natural capital and sustainable development.Conservation Biology.Vol. $6 \mathrm{~N}^{\circ} 1,46 \mathrm{p}$.

Daly H. 2002. Sustainable development: Definitions, Principles, Policies. World Bank, Washington, DC.Disponible en: http//millenniumindicators.un.org/unsd/envaccouting/ceea/archive/Framework/Daly_SD_ Def_Principles_Policies.PDF

Expocafe. Perú 2017. 7ma edición. Consultado en línea http://www.expocafeperu.com.pe/CafePeruano.php el 18 de febrero 2018.

Farfán F. 2007. Producción de café en sistemas agroforestales. En: Sistemas de producción y administración de cafetales. CENICAFE. Colombia. Cap. 8: 161-199

Gómez-Limón J.A. \& Arriaza M. 2011. Evaluación de la sostenibilidad de las explotaciones de olivar en Andalucía. 40p.

Harte MJ. 1995. Ecology, sustainability, and environment as capital. Ecologicaleconomics 15: $157-164$. 
Julca A., Meneses L., Rodriguez P., Bello S., Anahui J., Julca N., Borjas R., Crespo R., Santibañez R., Fundes G., Fundes D \& Reynoso A. 2009. Selección de fuentes naturales para la fertilización de café en el marco de una agricultura orgánica. [Comparación de Línea de base 2006 - Línea de Cierre, 2008]. Informe final de proyecto financiado por Incagro. Unalm-FDA.INIA-Café Perú- JNC. Lima. 32p.

Junta Nacional del Café. (2013). Quiénes somos. Recuperado de http://juntadelcafe.org.pe/quienes-somos

Martos J. 2004. Estadística: Conceptos, Práctica Aplicada y ejercicios. Departamento de Estadística y Organización de Empresas, Universidad de Córdoba, España

Merma I. \&Julca A. 2011. Evaluación y diseño de fincas en la selva alta bajo sistemas de cultivos prevalecientes en la Convención-Cusco. UNALM. Rev. Ecol. Apl. Vol. 11(1): 111.

Mendoza G. \&Prabhu R. 2000. Multiple criteria decision making approaches to assessing forest sustainability using criteria and indicators: a case study. ForestEcology and management 131: 107-126

Ministerio de Agricultura y Riego. (2013a, junio). Producción de principales cultivos, según región. Período: enero-junio. Años: 2012 y 2103. Estadística Mensual - Sistema Integrado de Estadística Agraria SIEA, 44-45. Recuperado de http://www.minag.gob.pe/portal/download/pdf/herramientas/boletines/boletineselectro nicos/estadisticaagrariamensual/2013/bemsa_junio13.pdf

Ministerio de Agricultura y Riego. (2003b). Caracterización de las zonas cafetaleras del Perú. Programa para el Desarrollo de la Amazonía. PROAMAZONÍA. Informe final. 117 pp. Recuperado de http://www.infocafes.com/descargas/biblioteca/94.pdf

Rodríguez C. 2002. diseño de indicadores de sustentabilidad para cuencas hidrográficas. Instituto Nacional de Ecología. 22pp.

Sarandón S. 2002. El desarrollo y uso de indicadores para evaluar la sustentabilidad de los agro ecosistemas. En Agroecología el camino hacia una agricultura sustentable. Ed. Científicas Americanas cap 20: 393-414. 450 p.

SarandónJ; Zuluaga S: CiezaR; Gómez C; JanjeticL y Negrete E. 2004. Evaluación de la sustentabilidad de sistemas agrícolas de fincas en Misiones, Argentina, mediante el uso de indicadores. RevistaAgroecología, Vol. 1: 19-28. 230 p.

SmithA y DumanskyJ. 1995. A framework for evaluating sustainable land management.Canadian Journal of SoilScience 75: 401-406. 410 p.

Velarde C. y Quiroz R. 1994. Análisis de Sistemas Agropecuarios. Uso de Métodos Biomatemáticos. CIRNMA-CONDESAN, La Paz, Bolivia. 238 p. 